\title{
QTL Mapping and Candidate Gene Analysis for Alkali Tolerance in Japonica Rice at the bud Stage Based on Linkage Mapping and Genome-Wide Association Study
}

Xianwei Li ${ }^{\dagger}$, Hongliang Zheng ${ }^{\dagger}$, Wenshen Wu, Hualong Liu, Jingguo Wang, Yan Jia, Jiaming Li, Luomiao Yang, Lei Lei, Detang Zou ${ }^{*}$ and Hongwei Zhao ${ }^{*}$ (i)

\begin{abstract}
Background: Salinity-alkalinity stress is one of the major factors limiting rice production. Damage caused by alkaline salt stress is more severe than that caused by neutral salt stress. Alkali tolerance at the bud stage in rice directly affects seedling survival and final yield when using the direct seeding cultivation model. However, genetic resources (QTLs and genes) for rice breeders to improve alkali tolerance are limited. In this study, we combined linkage mapping and a genome-wide association study (GWAS) to analyze the genetic structure of this trait in japonica rice at the bud stage.

Results: A population of 184 recombinant inbred lines (RILs) was utilized to map quantitative trait loci (QTLs) for the root length under control condition (RL), alkaline stress (ARL) and relative root length (RRL) at the bud stage. A major QTL related to alkali tolerance at the rice bud stage, qAT11, was detected on chromosome 11. Interestingly, a GWAS identified a lead SNP $\left(\mathrm{Chr}_{2} 21,999,659\right)$ in qAT11 that was significantly associated with alkaline tolerance. After filtering by linkage disequilibrium (LD), haplotype analysis, quantitative real-time $P C R$, we obtained three candidate genes (LOC_Os11937300, LOC_Os11937320 and LOC_Os11937390). In addition, we performed phenotype verification on the CRISPR/Cas9 mutant of LOC_Os11937390.

Conclusion: Based on these results, LOC_Os11937300, LOC_Os11937320 and LOC_Os11937390 were the candidate genes contributing to alkaline tolerance in japonica rice. This study provides resources for breeding aimed at improving rice responses to alkalinity stress.
\end{abstract}

Keywords: Japonica rice, The bud stage, Alkalinity tolerance, Linkage mapping, Genome-wide association study

\section{Background}

Soil salinity-alkalinity is one of the most important abiotic stresses limiting agricultural production. About 80 million hectares of irrigated soil worldwide are damaged by saline and alkaline stress, accounting for about $40 \%$ of the total irrigated area. With the increasing human

\footnotetext{
* Correspondence: zoudtneau@126.com; hongweizhao_cool@126.com ${ }^{+}$Xianwei Li and Hongliang Zheng contributed equally to this work. Key Laboratory of Germplasm Enhancement, Physiology and Ecology of Food Crops in Cold Region, Ministry of Education, Northeast Agricultural University, Harbin 150030, China
}

\section{Springer Open}

population, industrial modernization, and deterioration of the ecological environment, areas of reclamation are expanding and areas of soil salinity-alkalinity are increasing. It is estimated that by 2050 , soil salinization will destroy $50 \%$ of agricultural land in the world (Qadir et al. 2014). Rice (Oryza sativa L.) is one of the most important staple crops, feeding more than $60 \%$ of the population in China and providing $20 \%$ of energy per capita on a global scale (Cheng et al., 2007). It is also a salt-alkali-sensitive crop. Soil salinity-alkalinity can negatively affect rice seed germination, growth, seed

(c) The Author(s). 2020 Open Access This article is licensed under a Creative Commons Attribution 4.0 International License, which permits use, sharing, adaptation, distribution and reproduction in any medium or format, as long as you give appropriate credit to the original author(s) and the source, provide a link to the Creative Commons licence, and indicate if changes were made. The images or other third party material in this article are included in the article's Creative Commons licence, unless indicated otherwise in a credit line to the material. If material is not included in the article's Creative Commons licence and your intended use is not permitted by statutory regulation or exceeds the permitted use, you will need to obtain permission directly from the copyright holder. To view a copy of this licence, visit http://creativecommons.org/licenses/by/4.0/. 
maturation, the seed setting rate, and ultimately the rice yield (Zhu 2001; Shi et al. 2017). Therefore, studying salinity-alkalinity tolerance in rice has important practical significance for breeding tolerant cultivars and improving the utilization of saline-alkaline land ( $\mathrm{Li}$ et al. 2014).

Soluble salts in saline-alkali soil mainly include cations, such as $\mathrm{Na}^{+}, \mathrm{Ca}^{2+}, \mathrm{Mg}^{2+}$, and $\mathrm{K}^{+}$, and anions, such as $\mathrm{CO}_{3}{ }^{2-}, \mathrm{HCO}_{3}{ }^{-}, \mathrm{Cl}^{-}, \mathrm{SO}_{4}{ }^{2-}$, and $\mathrm{NO}_{3}{ }^{-}$, all of which come from neutral or alkaline salts. Stress caused by alkaline salts, such as $\mathrm{Na}_{2} \mathrm{CO}_{3}$ and $\mathrm{NaHCO}_{3}$, as well as high $\mathrm{pH}$ stress affect plants. Damage by alkaline salts is significantly greater than that caused by neutral salts, such as $\mathrm{NaCl}$ and $\mathrm{Na}_{2} \mathrm{SO}_{4}$, with distinct mechanisms of action (Guo et al. 2014). Recent research has increasingly focused on the serious environmental problem of alkaline stress. Soda saline-alkaline soil grassland in Northeast China has reached $70 \%$ of the total area and continues to expand. Therefore, the development of rice production on saline-alkali land has great significance for improving saline-alkali land and food security.

Owing to the low labor requirements and high efficiency, the direct seeding method has become an important cultivation model. Rice alkali tolerance at the bud stage is a major determinant of growth stability in saline-alkaline soil using the direct seeding cultivation model. During the planting season, rice seeds sensitive to alkali conditions at the bud stage exhibit low seedling rates and even seed death. Thus, improving the alkaline tolerance of rice at the bud stage is an important objective in rice breeding.

Salinity-alkalinity tolerance is a quantitative genetic characteristic that is controlled by multiple genes in rice (Qi et al. 2008). Extensive research has focused on the mechanism under lying salt tolerance in rice (Wang et al. 2011; Wang et al. 2012a; Sun et al. 2014). These studies have evaluated growth, morphology, physiology, and biochemistry, and some salt-tolerant genes have been identified by map-based cloning, such as SKC1 (Ren et al. 2005) and DST (Huang et al. 2009). However, less progress has been made in the localization of QTLs for alkali stress $\left(\mathrm{NaHCO}_{3}\right.$ or $\left.\mathrm{Na}_{2} \mathrm{CO}_{3}\right)$, and most of this research is in the stage of primary QTL mapping. For example, using $200 \mathrm{~F}_{2: 3}$ individuals, 13 and 6 QTLs related to the dead leaf rate and dead seedling rate under alkaline conditions, respectively, have been identified (Qi et al. 2008). A common QTL associated with the score of alkalinity tolerance and shoot $\mathrm{Na}^{+}$and $\mathrm{K}^{+}$concentrations in the rice seedling stage explained 13.36-13.64\% of phenotypic variation and was identified as OsIRO3 (Li et al. 2019). Cheng et al. (2008) detected 14 QTLs in $0.15 \% \mathrm{Na}_{2} \mathrm{CO}_{3}$ alkaline solution at the germination and early seedling stages. A previous study detected seven QTLs under alkaline stress, among which qSNC3 explained 21.24\% of the total phenotypic (Li et al. 2017). Additional genetic resources (QTLs/genes) that can be used by rice breeders to improve alkalinity tolerance are needed.

Bi-parental QTL mapping and GWAS are effective and precise tools for the detection of QTLs for complex traits (Wang et al. 2011; Kumar et al. 2015; Liang et al. 2015; Zheng et al. 2015; Shakiba et al. 2017; Shi et al. 2017). Combining the two methods can improve the breadth and accuracy of QTL detection. The combination of association analyses and linkage mapping for gene mining has been highly successful for studies of quantitative traits in rice. For example, linkage mapping and an association analysis have been used to detect a major QTL (LP1) controlling panicle length on chromosome 9 , which was further narrowed to a 90-kb region using a NIL-F2 population and analyzed by sequencing (Liu et al. 2016). A similar strategy was used to analyze the genetic structure of rice tolerance to aluminum toxicity, resulting in the identification of two QTLs (Famoso et al. 2011). These studies demonstrate the feasibility of identifying QTL/genes for alkali tolerance by combined linkage mapping and GWAS.

In this study, the root length under control condition (RL), alkaline stress (ARL) and relative root length (RRL) were used to assess the genetic basis of alkali tolerance in rice by linkage mapping and GWAS at the bud stage. A major QTL, qAT11, on chromosome 11 was detected by linkage mapping, and Chr11_21,999,659 within qAT11 was significantly associated with alkali tolerance, as determined by a GWAS. According to an LD analysis of the whole genome, a 218-kb region on chromosome 11 was selected for further analysis. Finally, we used haplotype analysis and gene expression analysis to obtained three candidate genes ( $L O C_{-} O s 11 g 37300, L O C_{-}$ Os11g37320 and LOC_Os11g37390) which were most likely involved in the regulation of alkali tolerance in rice.

\section{Results \\ Phenotypic Variation}

The means, standard deviations, and range of root lengths (RL), ARL, and RRL at the bud stage of RIL and natural populations are presented in Table S1. The mean ARLs were lower than RLs, indicating that alkali stress depressed the growth and development of japonica rice at the bud stage. Two parents of the RILs, Kongyu131 (KY131) and Xiaobaijingzi (XBJZ), showed different tolerances to alkali stress (Table S1). The ARL and RRL of KY131 were lower than those of XBJZ, indicating that $\mathrm{XBJZ}$ was more tolerant to alkali stress than KY131 at the bud stage. The mean RRL varied from 0.11 to 0.89 in the RIL population and from 0.25 to 0.94 in the natural population. The phenotypic values of RL, ARL and 
RRL in the two populations approximately followed a normal distribution, indicating that these indices are quantitative traits controlled by multiple factors (Fig. 1).

\section{Linkage Mapping for Alkali Tolerance at the Bud Stage} In total, 527 markers were used for QTL mapping of RL, ARL and RRL. Eight QTLs associated with the RL, ARL and RRL were detected on chromosomes 1, 4, 6, and 11 (Table 1 and Fig. S1), with LOD values of 2.76 to 7.55 and proportions of phenotypic variance explained from $4.03 \%$ to $18.75 \%$. qARL6 and $q R R L 6-2$ were located in the same interval and were considered the same QTL (named qAT6). qAT6 was located in the physical region between markers C6_10554837 and C6_19920701 and explained $6.35 \%-6.87 \%$ of the phenotypic variation. Similarly, $q A R L 11$ and $q R R L 11$ were considered the same QTL (named qAT11). qAT11 was located between markers C11_21685006 and C11_22560140 and explained $11.38 \%-18.75 \%$ of the phenotypic variation.

\section{GWAS for Alkali Tolerance-Related Traits in a Natural Population}

A GWAS was conducted with 788,369 SNPs obtained from previous studies in our laboratory (Li et al. 2019). Manhattan and quantile-quantile plots for the GWAS results are shown in Fig. 2. Nine lead SNPs significantly associated with RL, ARL and RRL are listed in Table 2. These SNPs were located on chromosomes 1, 5, 7, 11 and 12 with $R^{2}$ values ranging from $6.64 \%$ to $10.20 \%$. One lead SNP, Chr1_4,321,954, was located on chromosome 1 and was associated with ARL and RRL $\left(R^{2}=\right.$
8.82-10.20\%). The lead SNP, Chr11_21,999,659, associated with both ARL and RRL on chromosome 11 was located in qAT11 identified by the previous linkage mapping (Fig. $3 \mathrm{~b}$ and $\mathrm{c}$ ). According to a genome-wide LD decay analysis (Fig. 3a), we obtained a 218-kb region that was overlapping in linkage mapping and the GWAS. This interval is critical for alkali tolerance at the bud stage of rice and likely contains a candidate gene for this trait.

\section{Haplotype Analysis of Candidate Genes}

The 218-kb region contained 35 genes, including 17 functionally annotated genes, 9 genes encoding proteins with unknown functions, 2 genes encoding hypothetical proteins, and 7 genes encoding retrotransposons (Fig. 3d and Table S2). We conducted haplotype analysis on non-synonymous mutant SNPs of the exon region and SNPs of the promoter to identify important candidate genes in the above interval. Six genes (LOC_Os11g37230, LOC_Os11g37270, LOC_Os11g37300, LOC_Os11g37320, LOC_Os11g37340 and LOC_Os11g37390) were associated with significant differences in the RRL among different haplotypes (Fig. 4). Of the six genes, only $L O C_{-}$ Os11g37320 was divided into two haplotypes by SNP in the promoter region, and the other genes were divided into two or three haplotypes by non-synonymous mutation SNPs in the exon region.

Three haplotypes were found for LOC_Os11g37230 and LOC_Os11g37300. Haplotypes analysis revealed that significant difference for RRL was observed between hap1 (TT) and hap2 (GT) in LOC_Os11g37230 (Table 3
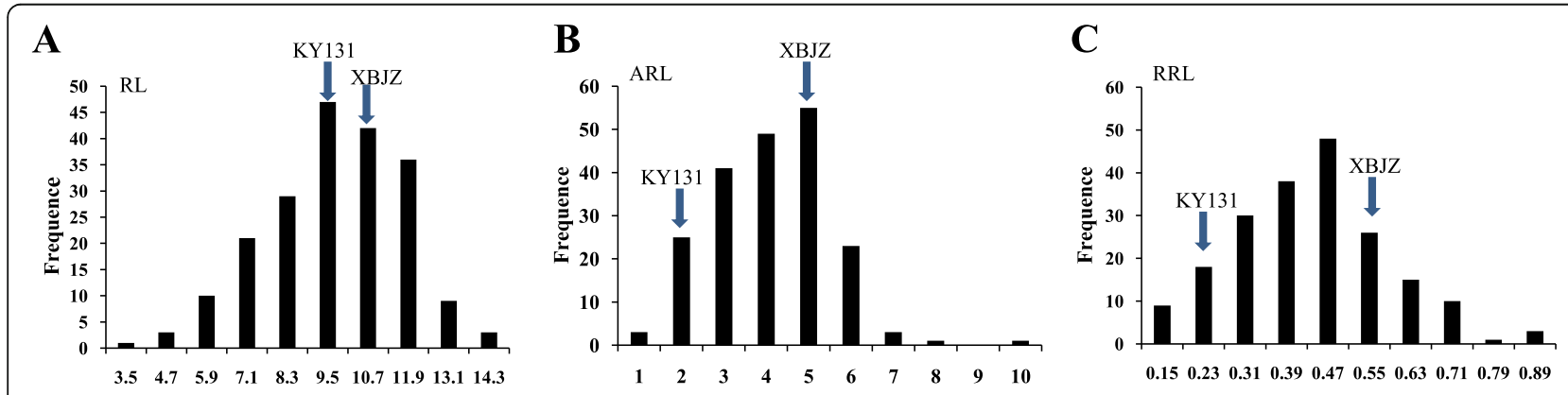

D
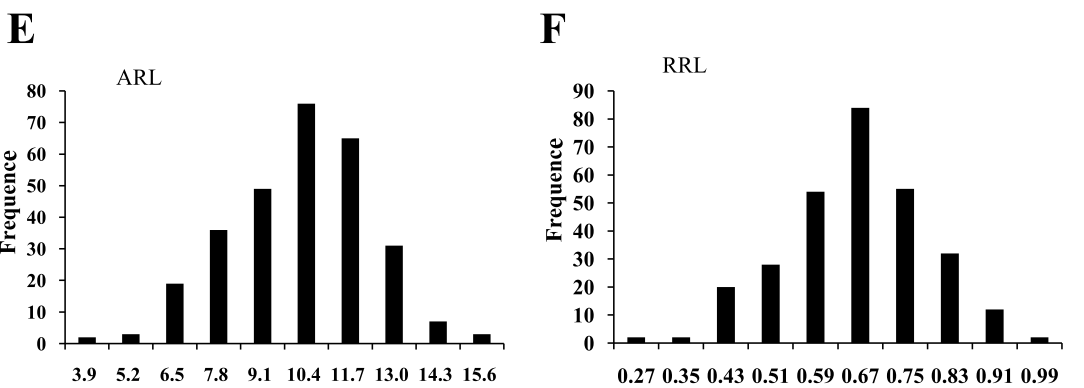

Fig. 1 Phenotypic variation in the $R L, A R L$, and $R R L$ in $R I L s$ and 295 japonica rice varieties. a, b and $\mathbf{c}$ represent the $R L, A R L$, and $R R L$ of $R I L s$, respectively; $\mathbf{d}$, e, and $\mathbf{f}$ represent the $R L, A R L$, and $R R L$ of natural populations, respectively 
Table 1 QTLs for RL, ARL and RRL based on the mean trait values of three replications in 184 RILs

\begin{tabular}{|c|c|c|c|c|c|c|c|c|}
\hline Traits & QTLS & Left Marker & Right Marker & Chr. & LOD & $R^{2}(\%)$ & Additive effect & QTL in previous study \\
\hline \multirow[t]{2}{*}{$\mathrm{RL}$} & qRL1 & C1_4655517 & C1_4984609 & 1 & 2.76 & 4.03 & 0.41 & \\
\hline & $q R L 4$ & C4_32090432 & C4_32195798 & 4 & 3.30 & 7.75 & -0.56 & \\
\hline \multirow[t]{3}{*}{ ARL } & qARL4 & C4_32090432 & C4_32195798 & 4 & 4.32 & 7.73 & -0.39 & $\begin{array}{l}\text { qIR4 (Wang et al. 2011) } \\
\text { qPH4 (Thomson et al. 2010) }\end{array}$ \\
\hline & qARL6 & C6_10554837 & C6_19920701 & 6 & 3.52 & 6.87 & 0.37 & qDRW6 (Wang et al. 2012b) \\
\hline & qARL11 & C11_21685006 & C11_22560140 & 11 & 6.03 & 11.38 & -0.48 & qSKC11 (Zang et al. 2008) \\
\hline \multirow[t]{3}{*}{ RRL } & qRRL6-1 & C6_4815311 & C6_4915271 & 6 & 3.15 & 4.05 & -3.03 & \\
\hline & aRRL6-2 & C6_10554837 & C6_19920701 & 6 & 3.96 & 6.35 & 3.72 & aDRW6 (Wang et al. 2012b) \\
\hline & qRRL11 & C11_21685006 & C11_22560140 & 11 & 7.55 & 18.75 & -6.45 & qSKC11 (Zang et al. 2008) \\
\hline
\end{tabular}

$\mathrm{R}^{2}(\%)$ : Phenotypic variance explained

and Fig. 4). Similarly, significant difference existed between hap1 (CTC) and hap2 (TCT) in LOC_Os11g37300 (Table 3 and Fig. 4). Two haplotypes were found for LOC_Os11g37270, LOC_Os11g37320, LOC_Os11g37340 and LOC_Os11g37390, significant differences for RRL between different haplotypes were observed for these four genes (Table 3 and Fig. 4).
Identification of Candidate Genes by Gene Expression and Sequence Analysis

According to the results of haplotype analysis, we evaluated the six genes by qRT-PCR in KY131 and XBJZ under alkali stress and control conditions (Fig. 5), and performed sequence analysis. Under normal condition, there was no significant difference in the expression

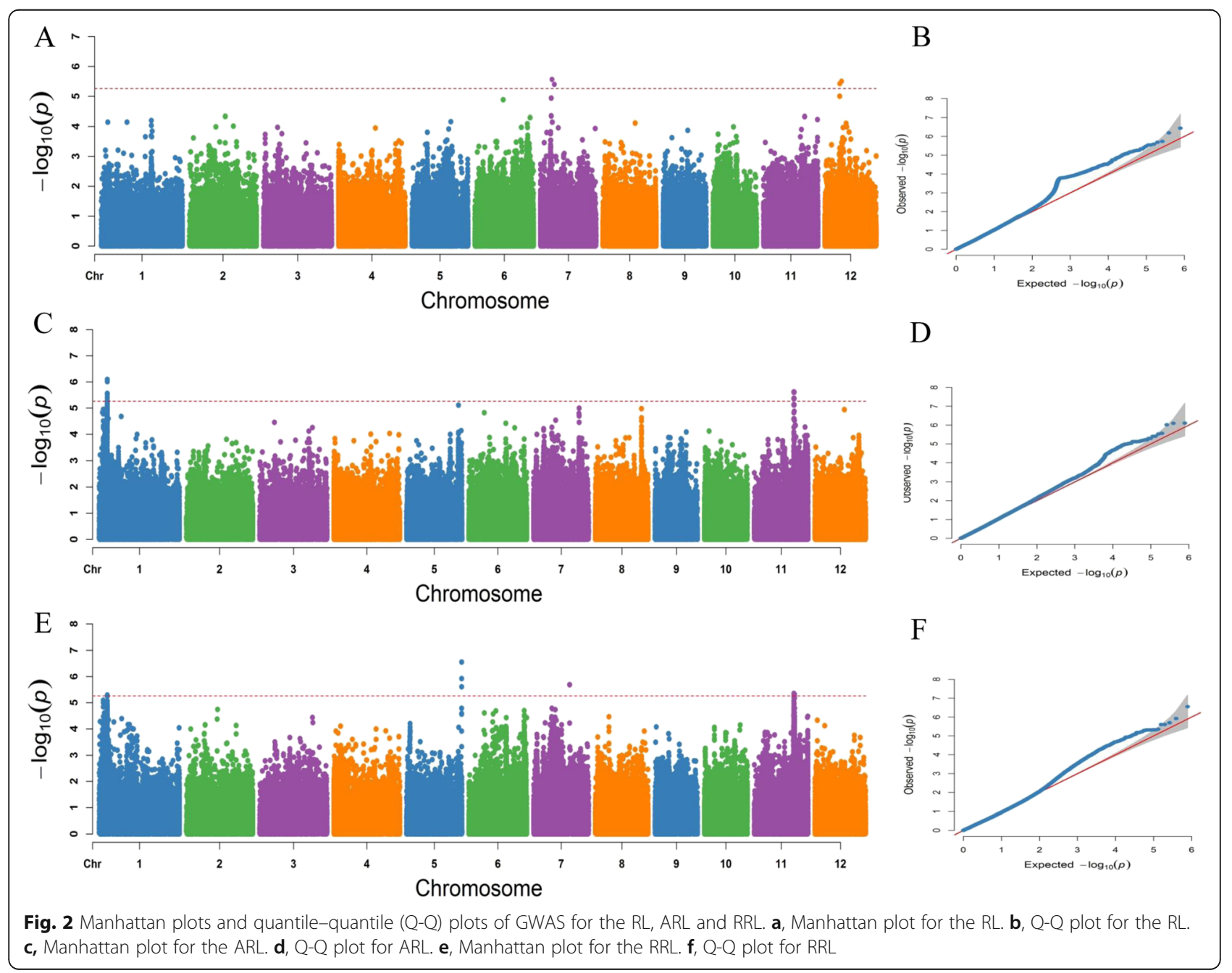


Table 2 Lead SNPS for RL, ARL and RRL identified by GWAS

\begin{tabular}{|c|c|c|c|c|c|c|}
\hline Traits & Lead SNP & Chromosome & Position & $P$ value & $R^{2}(\%)$ & QTL in previous study \\
\hline \multirow[t]{2}{*}{$\mathrm{RL}$} & Chr7_6,215,545 & 7 & $6,215,545$ & 5.07E-06 & 6.64 & \\
\hline & Chr12_ 7,813,381 & 12 & $7,813,381$ & $5.16 \mathrm{E}-06$ & 7.97 & \\
\hline \multirow[t]{2}{*}{$A R L$} & Chr1_4,321,954 & 1 & $4,321,954$ & $8.00 \mathrm{E}-07$ & 10.20 & qRL1 (Sabouri and Sabouri 2008) \\
\hline & Chr11__21,999,659 & 11 & $21,999,659$ & $9.74 \mathrm{E}-07$ & 10.05 & qSKC11 (Zang et al. 2008) \\
\hline \multirow[t]{5}{*}{ RRL } & Chr1_4,321,954 & 1 & $4,321,954$ & 5.11E-06 & 8.82 & qRL1 (Sabouri and Sabouri 2008) \\
\hline & Chr5_29,730,673 & 5 & $29,730,673$ & $2.84 \mathrm{E}-07$ & 9.59 & qRL5 (Sabouri and Sabouri 2008) \\
\hline & Chr7__19,513,614 & 7 & $19,513,614$ & $2.04 \mathrm{E}-06$ & 8.15 & qRL7 (Sabouri and Sabouri 2008) \\
\hline & Chr11_21,381,167 & 11 & $21,381,167$ & 4.43E-06 & 9.42 & \\
\hline & Chr11__21,999,659 & 11 & $21,999,659$ & 4.84E-06 & 10.19 & qSKC11 (Zang et al. 2008) \\
\hline
\end{tabular}

$\mathrm{R}^{2}(\%)$ : Phenotypic variance explained

levels of the six genes between KY131 and XBJZ. However, under alkalinity stress, three genes ( $L O C_{-}$ Os11g37300, LOC_Os11g37320, and LOC_Os11g37390) were differentially expressed between KY131 and XBJZ (Fig. 5). LOC_Os11g37390 showed higher expression levels in KY131 than in XBJZ. The opposite expression pattern was observed for LOC_Os11g37300 and LOC_ Os11g37320 (Fig. 5). In fact, the expression level of LOC_Os11g37390 in KY131 was nearly 10-fold higher than that in XBJZ after treatment with $40 \mathrm{mM} \mathrm{NaHCO}_{3}$, whereas only a 1-2-fold change was found for the other two genes. Of these three genes, LOC_Os11g37300 and $L O C \_O s 11 g 37390$ belong to the F-box gene family, which is influenced by salt stress (Jain et al. 2007).

We further sequenced the six genes (including gene and promoter region) in KY131 and XBJZ. LOC Os11g37390 of KY131 contained 1-bp insertion of a G base in the CDS region compared with the sequence in

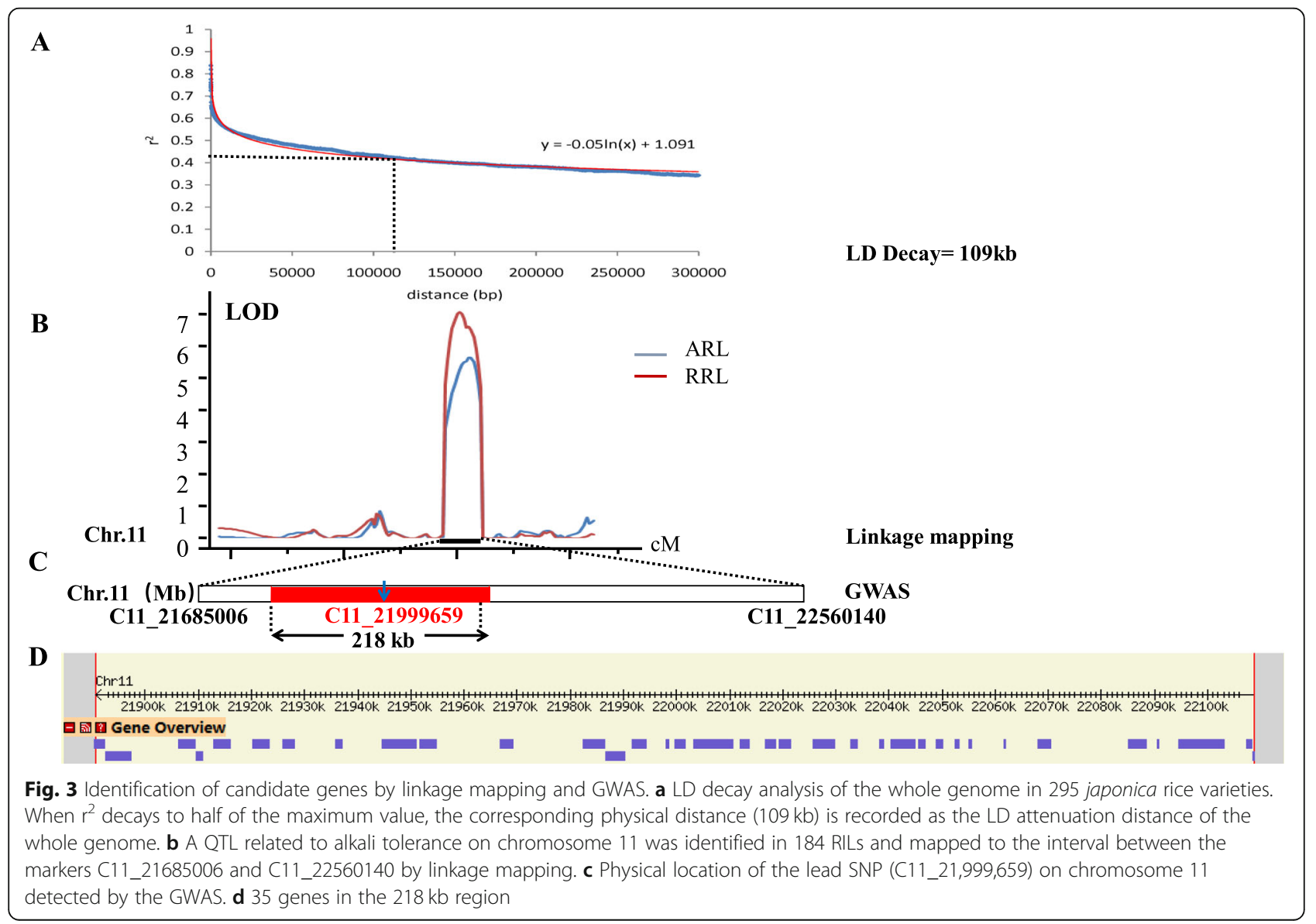




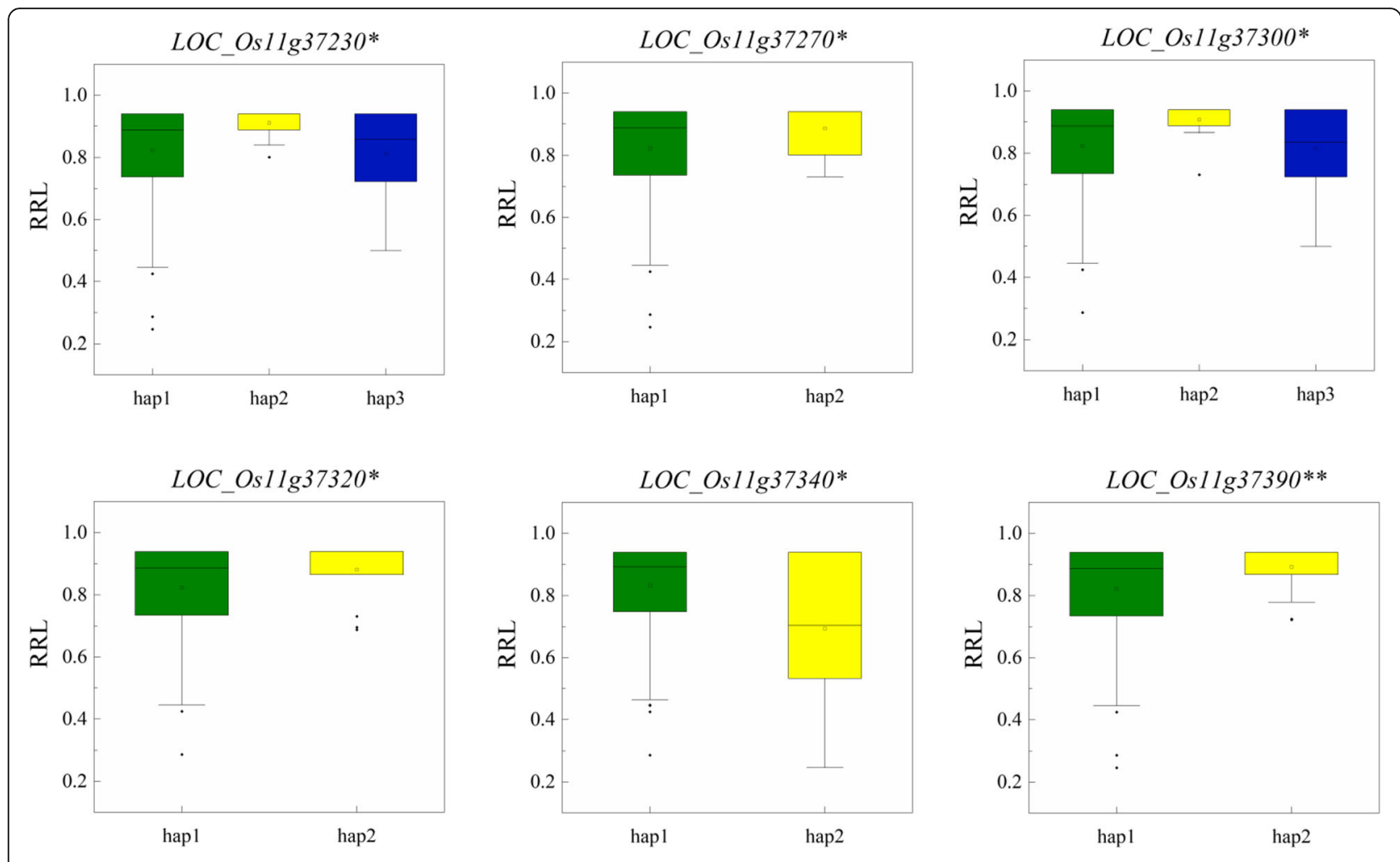

Fig. 4 Boxplots for RRL based on the haplotypes (Hap) for candidate genes; green, yellow and blue indicate the phenotypic result for hap1, hap2 and hap3, respectively (The ${ }^{*}$ and ${ }^{*}$ suggest significance of ANOVA at $P<0.05$ and $P<0.01$, respectively)

XBJZ. Other five genes exhibited no sequence differences between KY131 and XBJZ.

\section{LOC_Os11g37390 Mutant Is Sensitive to Alkali Stress}

To confirm the function of LOC_Os11g37390 in response to alkali stress, mutant named NPB-mutant, was obtained. The A base was inserted at 813 bp of the CDS region (Fig. 6a). The growth of NPB-mutant (Nipponbare background) was similar to that of Nipponbare; however, NPB-mutant was hypersensitive to alkali stress by $40 \mathrm{mM} \mathrm{NaHCO}$ treatment (Fig. 6b).

In the presence of $0 \mathrm{mM} \mathrm{NaHCO}$, no apparent difference was found between the NPB-mutant and Nipponbare plants roots, indicating that LOC_Os11g37390

Table 3 Candidate gene haplotype group and the composition of each haplotype SNP

\begin{tabular}{llll}
\hline Gene & hap1/ Number & hap2/ Number & hap3/ Number \\
\hline LOC_Os11g37230 & TT/261(XBJZ) & GT/10(KY131) & GA/10 \\
LOC_Os11g37270 & A/264(KY131) & G/14(XBJZ) & \\
LOC_Os11g37300 & CTC/250(KY131) & TCT/13(XBJZ) & CCC/12 \\
LOC_Os11g37320 & C/271(KY131) & T/15(XBJZ) & \\
LOC_Os11g37340 & CT/272(XBJZ) & TC/15(KY131) & \\
LOC_Os11g37390 & A/269(KY131) & T/17(XBJZ) & \\
\hline
\end{tabular}

(OsFBDUF54) had negligible effect on the growth of rice roots under normal conditions (Fig. 6b). However, after exposure to $40 \mathrm{mM} \mathrm{NaHCO}$ for 10 days, obvious differences were observed between the NPB-mutant and wildtype plants. The NPB-mutant plants were more sensitive to alkali stress than the wild-type plants. Under alkaline stress, the root lengths of the NPB-mutant plants were significantly shorter than those of the wild-type plants (Fig. 6b and c). Thus, LOC_Os11g37390 is the most likely candidate gene involved in the regulation of alkali tolerance in rice.

\section{Discussion}

Land and water productivity are seriously affected by saline and alkali stress, which obviously reduce crop production, such as soybean (Do et al. 2016), wheat (Francois et al. 1986), and rice (Liang et al. 2017). In contrast to salinity, alkalinity not only causes ionic toxicity in plants but also damages the stability of cells due to its high $\mathrm{pH}$, thus affecting the normal growth of plants (Chen et al. 2009). Many methods have been used to assess alkalinity tolerance in rice, and root length is a common phenotypic indicator (Liang et al. 2015). Plant roots are the main organs by which plants absorb water and nutrients. They have various biological functions, such as fixing above-ground parts, decomposing toxic 

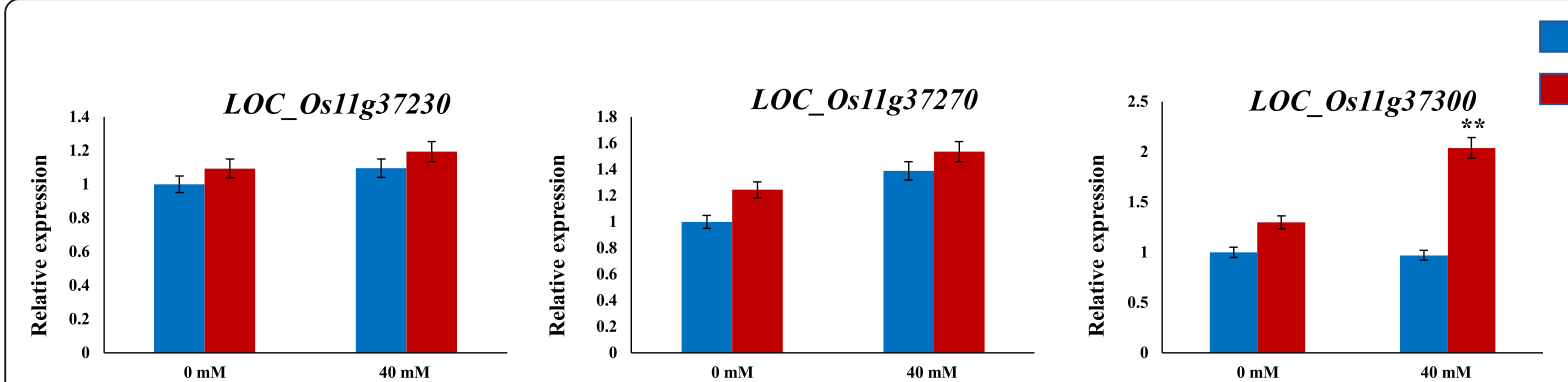

KY131
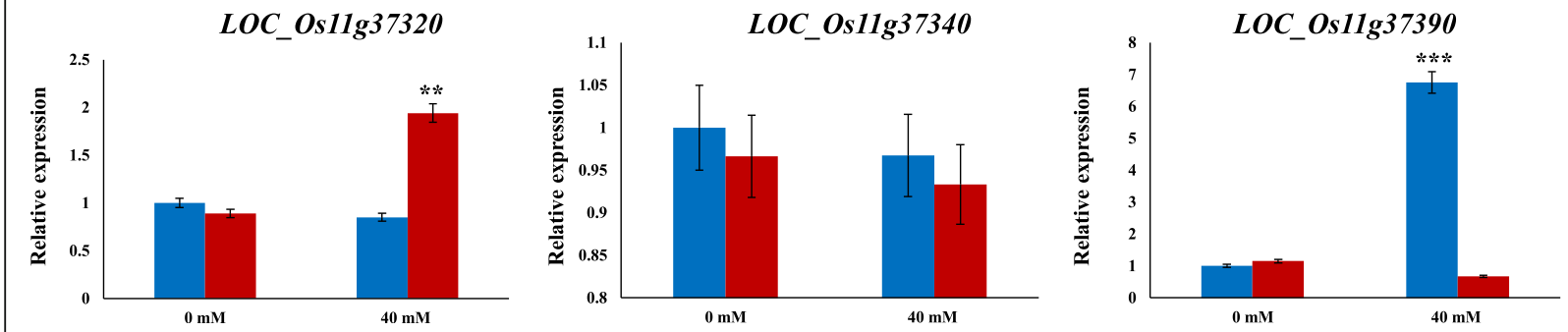

Fig. 5 Expression patterns of six genes under normal growth conditions and alkali stress $\left({ }^{* *} P<0.01 ;{ }^{* *} P<0.001\right.$; Student's $t$-test)

substances, and sensing environmental stimuli or stress (Kreszies et al. 2018). Analyses of root length at the bud stage in rice under alkaline stress provide a basis for breeding in saline-alkali land from the perspective of the root system, addressing the lack of rice germplasm resources for saline-alkali land.

Salinity-alkalinity tolerance is a highly complex trait in rice (Zhang et al. 2013). Many QTLs related to salt tolerance have been identified. However, few QTLs related to tolerance to alkali stress have been reported. Many different traits have been used as physical indexes for mapping QTLs for salt tolerance, and previously mapped loci overlapped with or were in close proximity to the loci identified in our study based on physical distance. A previous research detected three QTLs ( $q R L 1$, $q R L 5$, and $q R L 7$ ) on chromosomes 1, 5, and 7; the lead

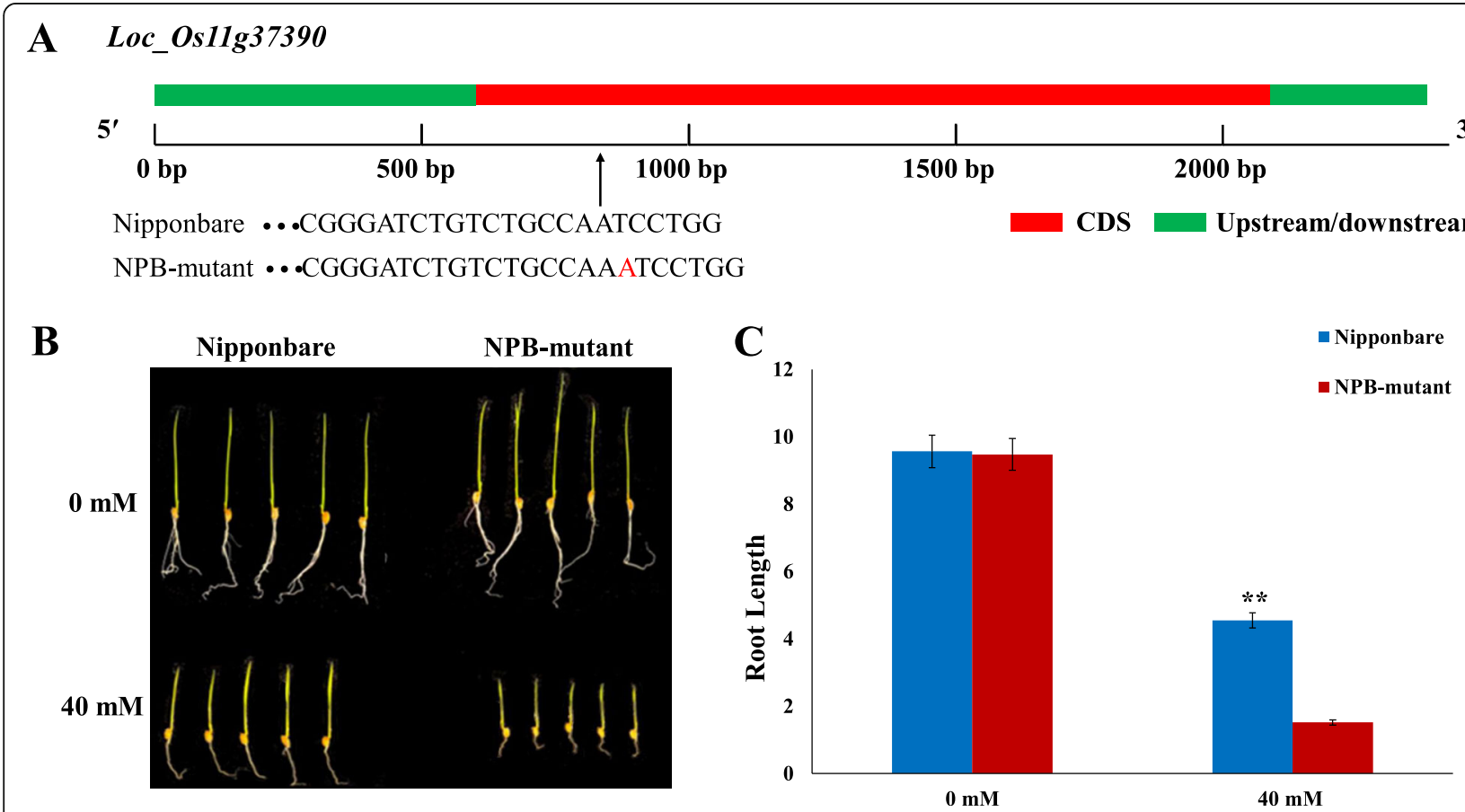

Fig. 6 a DNA sequence of LOC_Os11g37390 in Nipponbare and NPB-mutant. b Growth of NPB-mutant and control shoots treated with 0 or 40 $\mathrm{mM} \mathrm{NaHCO}_{3}$ for 10 days. c Significant differences in phenotypes between wild-type and NPB-mutant ( ${ }^{* *} P<0.01 ;$ Student's $t$-test) 
SNPs chr1_4,321,954, chr5_ 29,730,673, and chr7_ 19, 513,614 detected by our GWAS were located within or close to $q R L 1, q R L 5$, and $q R L 7$ (Sabouri and Sabouri 2008). It was found that qIR4 on chromosome 4 accounts for $22.9 \%$ of the phenotypic variation in the imbibition rate under salt stress and $q P H 4$ flanked by RM17391 and RM127 explains 25\% of the phenotypic variation for seedling height (Thomson et al. 2010; Wang et al. 2011). These two QTLs harbored qARL4 in our study by linkage mapping. $q D R W 6$, associated with dry root weight on chromosome 6 , contained qAT6, detected in our study by linkage mapping (Wang et al. 2012b). Another QTL, qSKC11 (Zang et al. 2008), contained $q A T 11$ and Chr11_21,999,659, identified in our study identified by linkage mapping and GWAS. These results supported the accuracy of our results.

In particular, we identified qAT11 and Chr11_21,999, 659 by linkage mapping and GWAS on chromosome 11 . According to the genome-wide LD decay of the GWAS (Fig. 3a), we identified a region of approximately $218 \mathrm{~kb}$ as a candidate region for further studies and this was the overlapping part in linkage mapping and the GWAS (Li et al. 2019). Based on haplotype analysis, six genes were associated with significant differences in the RRL among different haplotypes, on the basis of gene expression analysis, we obtained three candidate genes ( $L O C_{-}$ Os11g37300 named OsFBDUF53, LOC_Os11g37320 named LTPL79, and LOC_Os11g37390 named OsFBDUF54). Of the three candidate genes, LTPL79 is a protease inhibitor gene, and its function has not been preliminary studied, OsFBDUF53 and OsFBDUF54 belong to the F-box gene family, which is influenced by salt stress (Jain et al. 2007). Through sequence analysis of candidate genes we found that OsFBDUF54 of KY131 contained 1-bp insertion of a G base in the CDS region compared with the sequence in XBJZ. However, the other two genes (OsFBDUF53 and LTPL79) with different gene expression levels, even if there is no difference in nucleotide sequence, it can be a causal gene if the gene expression level is different. Through further integrated analysis, we selected OsFBDUF54 to conducted the preliminary phenotype verification of NPB-mutants, we identified a most likely candidate gene for alkalinity tolerance in rice.

F-box proteins, characterized by a conserved F-box motif of approximately 40 amino acids, represent one of the largest protein families, with about 700 members in Arabidopsis and rice (Gagne et al. 2002; Jain et al. 2007). In plants, only a small portion of F-box proteins have been studied; they have important roles in the regulation of various developmental processes and stress responses by integrating almost all phytohormone signaling pathways (Lechner et al. 2006; Dreher and Callis 2007; Zhang et al. 2008). Therefore, it is possible to speculate that they play important roles in a series of stress responses throughout the plant life cycle.

In the present study, the RRL of KY131 and XBJZ were 0.18 and 0.48 , respectively, and there were significant differences between the different haplotypes of OsFBDUF53, LTPL79 and OsFBDUF54. Furthermore, OsFBDUF54 expression was higher in the sensitive variety KY131 than in the alkali-tolerant variety XBJZ and the opposite expression pattern were observed for OsFBDUF53 and LTPL79 (Fig. 5). We further found that OsFBDUF54 of KY131 contained a 1-bp insertion in the CDS region relative to the sequence of XBJZ. So we assessed the response of OsFBDUF54 mutant to alkali stress and found the mutant was more sensitive than wild-type plants to alkali stress (Fig. 6c), this may explain the observed variation in alkali tolerance. MAF1 and OsFBDUF54 belong to the same gene family. According to previous analyses, the overexpression of MAF1 reduces abiotic stress tolerance and promotes root growth in rice (Yan et al. 2011). In the present study, OsFBDUF54 may be a regulator of alkali tolerance at the bud stage in rice, but additional data are needed to verify this conclusion. The overexpression and RNA interference of OsFBDUF54, OsFBDUF53 and LTPL79 will further clarify the role of the candidate genes in the alkali stress signaling cascade and will be a focus of future studies.

\section{Conclusions}

In the present study, RIL and natural populations were collected to evaluate the tolerance of alkalinity stress at the bud stage. A major QTL named qAT11 on chromosome 11 was detected by linkage mapping. Chr11_21, 999,659 located in qAT11 was significantly associated with alkali tolerance on chromosome 11, as determined by GWAS. According to an LD analysis of the whole genome, a 218-kb region was selected for further study. Based on haplotype analysis and qRT-PCR, LOC_ Os11g37300, LOC_Os11g37320 and LOC_Os11g37390 were the candidate genes contributing to alkaline tolerance in japonica rice. This study provides resources for breeding aimed at improving rice responses to alkalinity stress.

\section{Methods \\ Plant Material}

The linkage mapping population consisting of 184 RILs was derived from a cross between the japonica rice variety Kongyu131 (alkali-sensitive) and the upland rice variety Xiaobaijingzi (alkali-tolerant). The natural population for the GWAS comprised 295 japonica rice varieties, which were collected from the Liaoning, Jilin and Heilongjiang Provinces in China and other countries including the Republic of Korea, Japan, Russia and the Democratic People's Republic of Korea. All 295 japonica 
rice varieties belong to temperate japonica rice. All of the varieties in this population were selected from a previous study (Li et al. 2019).

\section{Evaluation of Alkali Tolerance at the Bud Stage}

Rice seeds were air-dried naturally, and kept at $55^{\circ} \mathrm{C}$ for 5 days to break dormancy. They were then surfacesterilized with $1 \%$ sodium hypochlorite solution for 10 $\mathrm{min}$, rinsed with sterile deionized water, and soaked in distilled water at $30^{\circ} \mathrm{C}$ in dark conditions for 3 days. When the seed bud length was greater than or equal to half the length of the seed, 50 uniform germinated seeds were divided into two portions. Each was seeded in a well of a thin polypropylene plate with a nylon mesh bottom, with a single seed sown per well. Finally, the two portions of germinated seeds were separately transferred to distilled water and a culture solution containing $40 \mathrm{mM} \mathrm{NaHCO}$. The seeds were placed in an incubator with a $28 / 21^{\circ} \mathrm{C}$ day/night cycle, natural sunlight, and $70 \% \mathrm{RH}$. The solution was changed every 2 days. After 10 days of alkaline stress, 10 seedlings with consistent growth were selected to measure the longest RL of the control and ARL. RRL was estimated to evaluate alkali tolerance. All phenotypes were tested in three replicates.

\section{QTL Mapping for Alkali Tolerance}

A genetic linkage map was constructed from 184 RILs using 527 bin markers obtained from $10 \mathrm{~K}$ Array genotyping by target sequencing (GBTS) by MOLBREEDING Biotechnology Company (Shijiazhuang, China). The constructed linkage map covered $1874.85 \mathrm{cM}$ of the rice genome with an average distance of $3.56 \mathrm{cM}$ between markers (Fig. S1). The QTL analysis was based on the arithmetic mean values from three replicates for each trait by the inclusive composite interval mapping (ICIM) method implemented in QTL IciMapping Ver.4.2 (http://www.isbreeding.net). The LOD score threshold for QTL identification was 2.5 for each trait, and the walking speed was $1 \mathrm{cM}$.

\section{GWAS for Alkali Tolerance}

In total, 788,369 SNPs with a minor allele frequency (MAF) of $\geq 5 \%$ and a missing rate of $\leq 20 \%$ were used to genotype 295 japonica rice accessions for GWAS. These were obtained by a preliminary study in our laboratory and the LD decay distance was $109 \mathrm{~kb}$ in the 295 japonica rice ( $\mathrm{Li}$ et al. 2019). GWAS was performed using the mixed linear model (MLM) implemented in TASSEL 5.0 (Bradbury et al. 2007). The threshold for the identification of SNPs significantly associated with traits was set to $P<5.46 \times 10^{-6}$, determined by genetic type 1 error calculator (GEC; http://statgenpro.psychiatry.hku.hk/ $\mathrm{gec} /$ ), which calculates the effective number of independent markers. To determine significant sites, if two or more significant SNPs were located in the same LD interval, then these SNPs were treated as the same QTL, and the SNP with the smallest $P$-value was taken as lead SNP. The contribution rate of this SNP was the contribution rate of the QTL. Manhattan and Q-Q plots were created using the $\mathrm{R}$ package 'qqman' from the GWAS results.

\section{Haplotype Analysis of Candidate Gene}

According to the LD decay analysis of the whole genome $($ LD decay $=109 \mathrm{~kb})$, haplotype analysis was performed on all genes in the $218 \mathrm{~kb}$ region. The non-synonymous mutation SNPs in the exon region of all genes in the interval were extracted from 3KRGP's RiceSNPSeekDatabase website (https://snp-seek.irri.org/). These SNPs were used to perform haplotype analysis of all genes in the interval using DnaSP software. For genes with no significant difference in RRL between different haplotypes ( $\geq 10$ materials), haplotype analysis was performed using SNPs in the promoter region $(1.5 \mathrm{~kb}$ before ATG).

\section{RNA Extraction and Quantitative Real-Time PCR Analysis}

After $72 \mathrm{~h}$ of alkalinity stress by $40 \mathrm{mM} \mathrm{NaHCO}$, rice roots from KY131 and XBJZ were sampled under alkaline and normal conditions. Total RNA was extracted from rice roots using the TranZol Up RNA Kit (TransGen Biotech, Beijing, China). Complementary DNA was synthesized from total RNA using the HiFiScript cDNA Synthesis Kit (Cwbio, Beijing, China). Quantitative realtime PCR (qRT-PCR) was performed using a Roche Light Cycler 2.10 system using $2 \times$ Fast qPCR Master Mix. Three biological replicates and three technical replicates were set for each sample. The mRNA levels of genes were determined relative to levels of the housekeeping gene Actin1 (Li et al. 2019) as an internal control. Relative gene expression levels were determined using the $2^{-\Delta \Delta C t}$ method (Livak and Schmittgen 2000). Data shown in figures and tables are mean values of three replicates.

\section{Candidate Gene Prediction, Sequencing, and Sequence Alignment}

Combining the linkage mapping and GWAS results, based on haplotype analysis results, six genes were selected as candidates. Then, the corresponding candidate genes were cloned by PCR and sequenced gene and promoter region in KY131 and XBJZ. The sequence alignment was generated using DNAMAN.

\section{OsFBDUF54 Mutant Plants}

The homozygous $T_{1}$ generation mutant seeds with Nipponbare background were obtained from BIOGLE 
GENETECH company (http://www.biogle.cn/) by CRIS PR/Cas9 method in October 2019. The $\mathrm{T}_{1}$ generation seeds were planted in Hainan of China, and the homozygous $\mathrm{T}_{2}$ generation seeds (named NPB-mutant) were obtained in March 2020 and they were used for alkali tolerance identification.

\section{Supplementary information}

Supplementary information accompanies this paper at https://doi.org/10. 1186/s12284-020-00412-5.

Additional file 1 : Fig. S1 Genetic linkage map and QTL mapping results.

Additional file 2 : Table S1. Descriptive statistics for root length under $0 \mathrm{mM} \mathrm{NaHCO}_{3}(\mathrm{C})$, and $40 \mathrm{NaHCO}_{3}$ (A) Summary of the trait means in the parents, 184 recombinant inbred lines (RILs), and 295 rice accessions.

Additional file $\mathbf{3}$ : Table S2 Summary of functional annotation results for genes in the candidate region on chromosome 11 .

Additional file 4 : Table S3 Primers used for qRT-PCR and relative expression in this study.

\section{Abbreviations}

GWAS: Genome-wide association study; RL: Root length; ARL: Root length under alkali stress; RRL: Relative root length; MAF: Minor allele frequency; QTL: Quantitative trait loci; SNP: Single-nucleotide polymorphism; LD: Linkage disequilibrium; RIL: Recombinant inbred line; KY131: Kongyu131; XBJZ: Xiaobaijingzi

\section{Acknowledgments}

We would thank to the the Natural Science Foundation Joint Guide Project of Heilongjiang (LH2019C035), the National Natural Science Foundation of China (31872884), and the "Young Talents" Project of Northeast Agricultural University (17QC02).

\section{Authors' Contributions}

$X W L, H L Z$, and HW Z conceived and designed the research. WS W, LM Y, $H L L$ and JG W participated in data analysis. DT $Z, L L, Y J$, and JM $L$ performed material development, sample preparation and data analysis. XW $L$ wrote the manuscript. HW Z corrected the manuscript. The final manuscript was read and approved by all authors.

\section{Funding}

This work was supported by the Natural Science Foundation Joint Guide Project of Heilongjiang (LH2019C035), the National Natural Science Foundation of China (31872884), and the "Young Talents" Project of Northeast Agricultural University (17QC02)

\section{Availability of Data and Materials}

The raw Illumina sequencing data from this study have been submitted to NCBI Sequence Read Archive (SRA) under the accession number PRJNA512109.

\section{Ethics Approval and Consent to Participate}

Not applicable.

\section{Consent for Publication}

Not applicable.

\section{Competing Interests}

The authors declare that they have no competing interests.
Received: 17 January 2020 Accepted: 8 July 2020

Published online: 16 July 2020

\section{References}

Bradbury PJ, Zhang Z, Kroon DE, Casstevens TM, Ramdoss Y, Buckler ES (2007) TASSEL: software for association mapping of complex traits in diverse samples. Bioinformatics 23:2633-2635

Chen W, Cui P, Sun H, Guo W, Yang C, Jin H, Fang B, Shi D (2009) Comparative effects of salt and alkali stresses on organic acid accumulation and ionic balance of seabuckthorn (Hippophae rhamnoides L.). Ind Crop Prod 30:351335

Cheng H, Jiang H, Xue D, Guo L, Zeng D, Zhang G, Qian Q (2008) Mapping of QTL underlying tolerance to alkali at germination and early seedling stages in rice. Acta Agron Sin 34:1719-1727

Cheng S, Cao L, Zhuang J, Chen S, Zhan X, Fan Y, Zhu D, Min S (2007) Super hybrid Rice breeding in China: achievements and prospects. J Integr Plant Biol 49:805-810

Do T, Chen H, Hien V, Hamwieh A, Yamada T, Sato T, Yan Y, Cong H, Shono M, Suenaga $\mathrm{K}$ (2016) $\mathrm{Ncl}$ synchronously regulates $\mathrm{Na}^{+}, \mathrm{K}^{+}$, and $\mathrm{Cl}^{-}$in soybean and greatly increases the grain yield in saline field conditions. Sci Rep 6: 19147

Dreher K, Callis J (2007) Ubiquitin, hormones and biotic stress in plants. Ann Bot 99:787-822

Famoso A, Zhao K, Clark R, Tung C-W, Wright M, Bustamante C, Kochian L, McCouch S (2011) Genetic architecture of aluminum tolerance in Rice (Oryza sativa) determined through genome-wide association analysis and QTL mapping. PLoS Genet 7:e1002221

Francois L, Maas E, Donovan T, Youngs V (1986) Effect of salinity on grain yield and quality, vegetative growth, and germination of semi-dwarf and durum Wheat1. Agron J 78:1053

Gagne J, Downes B, Shiu S, Durski A, Vierstra R (2002) The F-box subunit of the SCF E3 complex is encoded by a diverse superfamily of genes in Arabidopsis. Proc Natl Acad Sci U S A 99:11519-11524

Guo M, Wang R, Wang J, Hua K, Wang Y, Liu X, Yao S (2014) ALT1, a Snf2 family chromatin remodeling ATPase, negatively regulates alkaline tolerance through enhanced defense against oxidative stress in Rice. PLoS One 9: e112515

Huang X, Chao D, Gao J, Zhu M, Shi M, Lin H (2009) A previously unknown zinc finger protein, DST, regulates drought and salt tolerance in rice via stomatal aperture control. Genes Dev 23:1805-1817

Jain M, Nijhawan A, Arora R, Agarwal P, Ray S, Sharma P, Kapoor S, Tyagi A, Khurana J (2007) F-box proteins in Rice. Genome-wide analysis, classification, temporal and spatial gene expression during panicle and seed development, and regulation by light and abiotic stress. Plant Physiol 143:1467-1483

Kreszies T, Schreiber L, Ranathunge K (2018) Suberized transport barriers in Arabidopsis, barley and rice roots: from the model plant to crop species. J Plant Physiol 227:75-83

Kumar V, Singh A, Mithra S, Krishnamurthy S, Parida S, Jain S, Tiwari K, Kumar P, Rao A, Sharma S (2015) Genome-wide association mapping of salinity tolerance in rice (Oryza sativa). DNA Res 22:133-145

Lechner E, Achard P, Vansiri A, Potuschak T, Genschik P (2006) F-box proteins everywhere. Curr Opin Plant Biol 9:631-638

Li J, Pu L, Han M, Zhu M, Zhang R, Xiang Y (2014) Soil salinization research in China: advances and prospects. J Geogr Sci 24:943-960

Li N, Sun J, Wang J, Liu H, Zheng H, Yang L, Liang Y, Li X and Zou D (2017) QTL analysis for alkaline tolerance of rice and verification of a major QTL. Plant Breeding 136(6): 881-891.

Li N, Zheng H, Cui J, Wang J, Liu H, Sun J, Liu T, Zhao H, Lai Y, Zou D (2019) Genome-wide association study and candidate gene analysis of alkalinity tolerance in japonica rice germplasm at the seedling stage. Rice 12:24

Liang J, Qu Y, Yang C, Ma X, Cao G, Zhao Z, Zhang S, Zhang T, Han L (2015) Identification of QTLs associated with salt or alkaline tolerance at the seedling stage in rice under salt or alkaline stress. Euphytica 201:441-452

Liang Y, Sun J, Suo Y, Liu H, Wang J, Zheng H, Sun X, Zou D and University, N.A. (2017) QTL Mapping and QTL × Environment Interaction Analysis of Salt and Alkali Tolerance-Related Traits in Rice(Oryza sativa L.). Scientia Agricultura Sinica, 50(10): 1747-1762.

Liu E, Liu Y, Wu G, Zeng S, Thi T, Liang J, Liang Y, Dong Z, She D, Wang H, Zaid I and Hong D (2016) Identification of a Candidate Gene for Panicle Length in Rice (Oryza sativa L.) Via Association and Linkage Analysis. Frontiers in Plant Science 7, 596-596. 
Livak K and Schmittgen T (2001) Analysis of relative gene expression data using real-time quantitative $P C R$ and the $2^{-\Delta \Delta}$ method. Methods 25:402-408

Qadir M, Quillerou E, Nangia V, Murtaza G, Singh M, Thomas R, Drechsel P, Noble A (2014) Economics of salt-induced land degradation and restoration. Nat Res Forum 38:282-295

Qi D, Guo G, Lee M, Zhang J, Cao G, Zhang S, Suh S, Zhou Q, Han L (2008) Identification of quantitative trait loci for the dead leaf rate and the seedling dead rate under alkaline stress in rice. J Genet Genomics 35:299-305

Ren Z, Gao J, Li L, Cai X, Huang W, Chao D, Zhu M, Wang Z, Luan S, Lin H (2005) $A$ rice quantitative trait locus for salt tolerance encodes a sodium transporter. Nat Genet 37:1141-1146

Sabouri H, Sabouri A (2008) New evidence of QTLs attributed to salinity tolerance in rice. Afr J Biotechnol 7:4376-4383

Shakiba E, Edwards J, Jodari F, Duke S, Eizenga G (2017) Genetic architecture of cold tolerance in rice (Oryza sativa) determined through high resolution genome-wide analysis. PLoS One 12:e0172133

Shi Y, Gao L, Wu Z, Zhang X, Wang M, Zhang C, Zhang F, Zhou Y, Li Z (2017) Genome-wide association study of salt tolerance at the seed germination stage in rice. BMC Plant Biol 17:92

Sun J, Zou D, Luan F, Zhao H, Wang J, Liu H, Xie D, Su D, Ma J, Liu Z (2014) Dynamic QTL analysis of the $\mathrm{Na}^{+}$content, $\mathrm{K}^{+}$content, and $\mathrm{Na}^{+} / \mathrm{K}^{+}$ratio in rice roots during the field growth under salt stress. Biol Plant 58:689-696

Thomson M, de Ocampo M, Egdane J, Rahman M, Sajise A, Adorada D, Tumimbang-Raiz E, Blumwald E, Seraj Z, Singh R, Gregorio G and Ismail A (2010) Characterizing the Saltol quantitative trait locus for salinity tolerance in Rice. Rice 3, 148-160

Wang Z, Chen Z, Cheng J, Lai Y, Wang J, Bao Y, Huang J, Zhang H (2012a) QTL analysis of $\mathrm{Na}^{+}$and $\mathrm{K}^{+}$concentrations in roots and shoots under different levels of NaCl stress in rice (Oryza sativa L.). PLoS One 7:e51202

Wang Z, Cheng J, Chen Z, Huang J, Bao Y, Wang J, Zhang H (2012b) Identification of QTLs with main, epistatic and QTL x environment interaction effects for salt tolerance in rice seedlings under different salinity conditions. Theor Appl Genet 125:807-815

Wang Z, Wang J, Bao Y, Wu Y, Zhang H (2011) Quantitative trait loci controlling rice seed germination under salt stress. Euphytica 178:297-307

Yan Y, Chen X, Yang K, Sun Z, Fu Y, Zhang Y, Fang R (2011) Overexpression of an $\mathrm{F}$-box protein gene reduces abiotic stress tolerance and promotes root growth in rice. Mol Plant 4:190-197

Zang J, Sun Y, Wang Y, Yang J, Li F, Zhou Y, Zhu L, Jessica R, Mohammadhosein F, Xu J, Li Z (2008) Dissection of genetic overlap of salt tolerance QTLs at the seedling and tillering stages using backcross introgression lines in rice. Sci In China Series C-Life Sci 51:583-591

Zhang Y, Lin X, Ou X, Hu L, Wang J, Yang C, Wang S, Liu B (2013) Transcriptome alteration in a rice introgression line with enhanced alkali tolerance. Plant Physiol Biochem 68:111-117

Zhang Y, Xu W, Li Z, Deng X, Wu W, Xue Y (2008) F-box protein DOR functions as a novel inhibitory factor for Abscisic acid-induced Stomatal closure under drought stress in Arabidopsis. Plant Physiol 148:2121-2133

Zheng H, Zhao H, Liu H, Wang J, Zou D (2015) QTL analysis of $\mathrm{Na}^{+}$and $\mathrm{K}^{+}$ concentrations in shoots and roots under $\mathrm{NaCl}$ stress based on linkage and association analysis injaponicarice. Euphytica 201:109-121

Zhu J (2001) Plant salt tolerance. Trends Plant Sci 6:66-71

\section{Publisher's Note}

Springer Nature remains neutral with regard to jurisdictional claims in published maps and institutional affiliations.

\section{Submit your manuscript to a SpringerOpen ${ }^{\circ}$ journal and benefit from:}

- Convenient online submission

- Rigorous peer review

- Open access: articles freely available online

- High visibility within the field

- Retaining the copyright to your article

Submit your next manuscript at $\boldsymbol{\nabla}$ springeropen.com 